\title{
Rising from the crypt: decreasing DNA methylation during differentiation of the small intestine
}

\author{
Sean M Cullen and Margaret A Goodell* \\ Please see related research article: http://genomebiology.com/2013/14/5/R50
}

\begin{abstract}
The differentiation of intestinal stem cells involves few DNA methylation changes, assayed by bisulfite sequencing, in contrast to other adult somatic stem cell hierarchies.

Keywords: DNA methylation, Somatic stem cell differentiation, Lgr5, WGBS, Intestinal stem cell
\end{abstract}

\section{DNA methylation and its influence upon cell fate}

DNA methylation at CpG dinucleotides is the prototypical mode of epigenetic regulation, enabling stable but reversible gene repression. DNA methylation is highly dynamic in early embryonic development, with a near-complete erasure after fertilization, followed by a wave of de novo methylation around the time of implantation. As development progresses and lineage choice decisions are made, many loci undergo cell-type-specific DNA methylation changes, as the chosen gene expression programs are fixed [1]. Thereafter, DNA methylation is thought to be highly stable, except during oncogenic transformation, where methylation is well known to be highly aberrant [2]. With the current surge of interest in stem cell biology, the question arises as to how, and to what degree, DNA methylation during stem cell differentiation can influence lineage fate decisions, which represent developmental decisions that continue to occur throughout adult life. Understanding how DNA methylation changes between somatic progenitors and their progeny could lend insight into the mechanisms of development and epigenetic regulation. With the advent of large-scale, genome-wide bisulfite sequencing, the generation of DNA methylation profiles of purified cell populations at single-base resolution has been facilitated. A study by Kaaij et al. in this issue of Genome Biology [3]

*Correspondence: Goodell@bcm.edu

Stem Cells and Regenerative Medicine Center \& Program in Developmental Biology, Baylor College of Medicine, Houston, TX 77030, USA is the first to examine at high resolution the methylation changes that take place during the differentiation of intestinal stem cells (ISCs), a well-characterized somatic stem cell population.

\section{Paucity of methylation changes found between intestinal stem cells and their progeny}

Utilizing a transgenic green fluorescent protein (GFP) mouse line that fluorescently labels the ISC population and its downstream progeny with graded levels of fluorescence [4], Kaaij et al. [3] isolated ISCs as GFPhigh (stem cells in the base of the intestinal crypt), transit amplifying cells as GFPlow (rapidly dividing cells moving up the intestinal crypt), and terminally differentiated (villus) populations in order to capture the differentiation hierarchy. Whole-genome bisulfite sequencing (WGBS) was then performed on each of the three isolated intestinal cell populations. The generation of this dataset alone is significant, as genome-wide studies to deduce the impact of DNA methylation on normal differentiation of adult somatic tissue systems are relatively recent and few in number [5-7].

Surprisingly, WGBS revealed only minor alterations in DNA methylation during the differentiation from an ISC to the mature progeny of the intestinal villus. Of the few differentially methylated regions (DMRs) observed during differentiation, most were in loci identified by histone marks as gene-regulatory domains, specifically enhancers. The vast majority of these DMRs (43/50) were found to lose methylation during differentiation. The authors confirmed that a number of the hypomethylated DMRs physically interact with genes upregulated upon differentiation, suggesting that there is a mechanistic link between altering DNA methylation status and the progression of differentiation. The remaining seven DMRs exhibited hypermethylation upon differentiation, but these shifts did not correlate with changes in gene expression. These findings, combined with the observed low expression of the de novo DNA methyltransferases (Dnmt3a and Dnmt3b) in ISCs, led the authors to propose that de novo DNA methylation is not relevant in ISC differentiation. 


\section{Increased DNA methylation dynamics observed in other stem cell populations}

The limited number and direction of DNA methylation changes that Kaaij et al. observed were unexpected in the context of previous studies on embryonic stem cell (ESC) and somatic progenitor cell differentiation. In a study of genome-wide DNA methylation changes of ESCs undergoing differentiation to neural progenitor cells (NPCs) in vitro, the majority of CpGs with differential methylation gained methylation, with approximately $8 \%$ of CpGs gaining and only approximately $2 \%$ of CpGs losing methylation; this suggests active de novo methylation activity during ESC differentiation [8].

In the mouse hematopoietic system, which has a welldescribed hierarchy of stem, progenitor and differentiated cells, relatively homogenous cell populations can be purified and analyzed for their methylation patterns. In recent studies using reduced representation bisulfite sequencing (RRBS) or comprehensive high-throughput arrays for relative methylation (CHARM), hematopoietic progenitors and their differentiated progeny were found to exhibit a large degree of differential methylation, which correlated with lineage-specific gene expression changes $[5,6]$. In addition, application of WGBS to human hematopoietic progenitors showed that they possess partially methylated regions, believed to be similar in function to poised bivalent domains in ESCs. These sites of intermediate methylation were then found to either gain or lose methylation in B cells, and vice versa in neutrophils, suggesting that the DNA methylation changes were lineage specific [7]. Moreover, a role for $d e$ novo methylation during hematopoietic stem cell (HSC) differentiation was directly shown using conditional knockout mice, in which the differentiation of Dnmt3amutant HSCs was significantly impeded after bone marrow transplantation. RRBS analysis showed that Dnmt3a functions, at least in part, to ensure the stem cell program is shut down during differentiation [9].

While not all of the above-described studies used highly purified populations, and the resolution of the methylation profiling could be improved, the overall message that hematopoietic differentiation is accompanied by dynamic methylation changes is likely to prevail. Additionally, DNA methylation changes accompanying somatic stem cell differentiation are not limited to the hematopoietic system, but have been readily found within the skin lineage differentiation program. RRBS analysis of skin progenitors and their mature progeny identified over 1,700 DMRs that either gained (269 regions) or lost (1487 regions) methylation during differentiation [5]. This is quite distinct from the very limited changes in the intestine reported by Kaaij and colleagues. Furthermore, the previously described RRBS analysis of in vivo hematopoietic and skin progenitors and their progeny found a number of shared DMRs that either gained (258) or lost (248) methylation during differentiation [5] that was significantly larger than the total number of DMRs found during ISC differentiation (50) [3]. A number of these shared DMRs were characterized by both open chromatin signatures and DNAasehypersensitive sites found in other lineages (brain, heart and kidney), leading to a prediction of shared epigenetic regulatory patterns between somatic stem cell populations undergoing differentiation [5]. While the possibility of such overarching patterns is enticing, further characterization of other somatic stem cell populations at high resolution and stem cell purity is still required. Studies at a depth comparable to that of this article will be necessary to make such generalizations confidently.

\section{Are there additional roles for DNA methylation in intestinal differentiation?}

This study by Kaaij et al. [3] stands out as one of few to examine genome-wide DNA methylation changes in normal cells directly isolated from fresh tissue as opposed to after in vitro culture. This approach avoids the potentially confounding result of aberrant DNA methylation from in vitro culture, which has been shown to lead to gradual hypermethylation [8]. With such a tiny number of identified methylation changes, however, one has to conjecture whether the authors could have missed something. One potential caveat is in the approach the authors used to isolate intestinal cell populations, which might lead to some heterogeneity in the cell populations examined. While GFPhigh cells in this transgenic mouse have been shown to represent pure stem cell populations [4], there is a greater degree of heterogeneity within the villus-defined population. The villus, while comprising primarily absorptive enterocytes (approximately 90\%), also contains mucosecreting goblet cells and various subtypes of enteroendocrine cells (approximately 1\%) [10]. This heterogeneity could possibly dampen the ability to identify subtle methylation differences among the cell populations analyzed, but is unlikely to change the overall conclusions.

In addition, the authors posit that, owing to both low expression of de novo methyltransferases (Dnmt3a and Dnmt3b) and the predominance of hypomethylated DMRs, active de novo methylation might not serve an important role in differentiation of the small intestine. Could DNA methylation changes, however, influence the decision between secretory or absorptive cell types, similar to the influence DNA methylation has on myeloid versus lymphoid fate decisions [6,7]? In addition, the analysis of adult intestinal somatic stem cell differentiation does not preclude a possible role for de novo DNA methylation earlier in intestinal development. It might be worthwhile examining both methylation and gene expression changes 
between purified, mature intestinal cell populations sourced at different developmental time-points to address these questions.

Overall, this study is a valuable addition to the growing body of data uncovering roles for DNA methylation in the differentiation of known normal somatic stem cells. With some similarities, but more differences, to other somatic stem cell populations previously studied, future studies will be needed to discern whether common epigenetic changes during somatic stem cell differentiation exist, and to test how such distinctions influence the specific differentiation process of the stem cells of each tissue system.

\section{Abbreviations}

CHARM, comprehensive high-throughput arrays for relative methylation; DMR, differentially methylated region; ESC, embryonic stem cell; HSC, hematopoietic stem cell; ISC, intestinal stem cell; NPC, neural progenitor cell: RRBS, reduced representation bisulfite sequencing; WGBS, whole-genome bisulfite sequencing.

\section{Competing interests}

The authors declare they have no competing interests.

Published: 28 May 2013

\section{References}

1. Smith ZD, Meissner A: DNA methylation: roles in mammalian development. Nat Rev Genet 2013, 14:204-220.

2. Herman JG, Baylin SB: Gene silencing in cancer in association with promoter hypermethylation. N Engl J Med 2003, 349:2042-2054.
3. Kaaij LJT, van de Wetering M, Fang F, Decato B, Molaro A, van de Werken HJG, van Es JH, Schuijers J, de Wit E, de Laat W, Hannon GJ, Clevers HC, Smith AD, Ketting RF: DNA methylation dynamics during intestinal stem cell differentiation reveals enhancers driving gene expression in the villus. Genome Biol, 14:RXX

4. Barker N, van Es JH, Kuipers J, Kujala P, van den Born M, Cozijnsen M, Haegebarth A, Korving J, Begthel H, Peters PJ, Clevers H: Identification of stem cells in small intestine and colon by marker gene Lgr5. Nature 2007, 449:1003-1007.

5. Bock C, Beerman I, Lien W-H, Smith ZD, Gu H, Boyle P, Gnirke A, Fuchs E, Rossi DJ, Meissner A: DNA methylation dynamics during in vivo differentiation of blood and skin stem cells. Mol Cell 2012, 47:633-647.

6. Ji H, Ehrlich LIR, Seita J, Murakami P, Doi A, Lindau P, Lee H, Aryee MJ, Irizarry RA, Kim K, Rossi DJ, Inlay MA, Serwold T, Karsunky H, Ho L, Daley GQ, Weissman IL, Feinberg AP: Comprehensive methylome map of lineage commitment from haematopoietic progenitors. Nature 2011, 467:338-342.

7. Hodges E, Molaro A, Dos Santos CO, Thekkat P, Song Q, Uren PJ, Park J, Butler J, Rafii S, McCombie WR, Smith AD, Hannon GJ: Directional DNA methylation changes and complex intermediate states accompany lineage specificity in the adult hematopoietic compartment. Mo/ Cell 2011, 44:17-28.

8. Meissner A, Mikkelsen TS, Gu H, Wernig M, Hanna J, Sivachenko A, Zhang X, Bernstein BE, Nusbaum C, Jaffe DB, Gnirke A, Jaenisch R, Lander ES: Genomescale DNA methylation maps of pluripotent and differentiated cells. Nature 2008, 454:766-771.

9. Challen GA, Sun D, Jeong M, Luo M, Jelinek J, Berg JS, Bock C, Vasanthakumar A, Gu H, Xi Y, Liang S, Lu Y, Darlington GJ, Meissner A, Issa JP, Godley LA, Li W, Goodell MA: Dnmt3a is essential for hematopoietic stem cell differentiation. Nat Genet 2011, 44:23-31.

10. Barker $N$, van de Wetering M, Clevers $H$ : The intestinal stem cell. Genes Dev 2008, 22:1856-1864.

doi:10.1186/gb-2013-14-5-116

Cite this article as: Cullen SM, Goodell MA: Rising from the crypt: decreasing DNA methylation during differentiation of the small intestine. Genome Biology 2013, 14:116. 